\title{
Bending Vibration Suppression of Railway Vehicle Carbody with Piezoelectric Elements (Experimental Results of Excitation Tests with a Commuter Car)*
}

\author{
Tadao TAKIGAMI** ${ }^{* *}$ and Takahiro TOMIOKA** \\ ** Vehicle Noise and Vibration Lab., Vehicle Structure Technology Division, \\ Railway Technical Research Institute \\ 2-8-38 Hikari-Cho, Kokubunji, Tokyo, 185-8540, Japan \\ E-mail: takigami@ rtri.or.jp, tomioka@ rtri.or.jp
}

\begin{abstract}
This paper describes the application of piezoelectric elements to suppress the bending vibration of a railway vehicle carbody. The elements are electrically connected to external shunt circuits. The authors carried out stationary excitation tests by using a commuter car with damper units consisting of piezoelectric elements and attachment devices. Two types of shunt circuits, an inductor and a resistor in series $(L-R$ circuit) and a capacitor with a negative value and a resistor (negative $C-R$ circuit), are implemented in the tests. The test results indicate that the damper units reduce the frequency response gain between excitation force and acceleration on the floor at the natural frequency up to approximately $30 \%$.
\end{abstract}

Key words : Piezoelectric Element, Railway Vehicle, Commuter Car, Bending Vibration, Ride Comfort, Electric Shunt Circuit

\section{Introduction}

Recently manufactured railway vehicles are increasingly lighter in weight. This fact contributes to increase of train speed, the saving of energy, etc. On the other hand, there are cases in which the vertical bending vibration of carbodies becomes conspicuous, such vibration often occurs in a frequency range to which passengers aboard trains are very sensitive. Since the vibration causes ride comfort to decline, effective measures to suppress it are called for.

Several methods have already been proposed to suppress such bending vibration, for example, by attaching damping layers consisting of rubbers and carbon fiber reinforced plastics (CFRPs) ${ }^{(1)}$, optimizing specifications of connecting elements between a carbodies and trucks $^{(2)}$, utilizing a tuned mass damper, or applying active and semi-active vibration control systems $^{(3)(4)}$. However, only a few of these are now being put into practical use because of the problems including high cost, heavy weight, low efficiency and/or low reliability. Therefore, it is essential to develop new techniques to reduce vibration more effectively with reasonable costs and weights.

The goal of this study is to reduce the bending vibration of railway vehicle carbodies by applying a vibration suppression technique that utilizes piezoelectric elements, such as PZTs, shunted by an external electric circuit. This technique was originally presented as explicit in the Ref. (5), and have been extensively studied ${ }^{(6)}$. Since its application was mainly to smallsize structures ${ }^{(7)(8)}$, however, it is expected to deal with a considerable number of problems in applying to such large structures as railway vehicle carbodies.

The authors have therefore conducted investigations to reduce the bending vibration of a primitive rectangular tube structure with the length of $3 \mathrm{~m}^{(9)}$ and a scale model of a Shinkansen vehicle with the length of $4.9 \mathrm{~m}^{(10)}$ as the primary steps to realize the foregoing goal. And 
then, in this paper, this vibration suppression technique has been applied to an actual railway vehicle as the next step of our study. The authors performed stationary excitation tests for a commuter vehicle, whose carbody is $19.5 \mathrm{~m}$ long, to investigate the performance of vibration suppression.

\section{The Railway Vehicle Carbody to Be Tested}

\subsection{Outline of the Carbody}

Figure 1 shows the railway vehicle carbody to be tested in this study. This is a test vehicle belonging to the Railway Technical Research Institute (RTRI), and has almost identical carbody structures to those in the current commercial vehicles. The carbody shell is of a stainless steel structure, which is dominant for commuter railway vehicles in Japan. The test vehicle has passenger seats, while it does not provided with electrical service equipments such as air conditioning or lightings facilities. The length of the carbody is $19.5 \mathrm{~m}$; the width, $2.95 \mathrm{~m}$; the height, $2.67 \mathrm{~m}$, and the mass of the carbody is approximately 10.7 tons.

\subsection{Vibration Characteristics of the Carbody}

In order to obtain the modal vibration characteristics of the carbody without piezoelectric elements, we carried out preliminary stationary excitation tests. An electrodynamic exciter is applied at the longitudinal center on the side beam of the floor through a driving rod to excite the carbody in the vertical direction.

We measured the excitation force with a load cell attached between the driving rod and the carbody, as well as the response vibration accelerations with acceleration pickups stuck on the floor, roof and side panel of the carbody. The input to the exciter is chosen as the band random signal that has an uniform frequency component in the range 5-20 Hz. Figure 2 shows the arrangements of the electrodynamic exciter. Figure 3 shows the various mode shapes together with their natural frequencies obtained by the stationary excitation test. The thin black lines show the undeformed shapes which links the original positions of acceleration pickups and the thick blue lines express the vibration shapes corresponding to the eigen modes.

As shown in the figure, six bending modes are identified and these mode shapes are complex; the vibration shapes and/or phase of roof and floor are different each other in those bending modes. Moreover, the frequency range of these six modes is relatively narrow and these frequencies are in the high sensitive region of humans for vertical vibration.

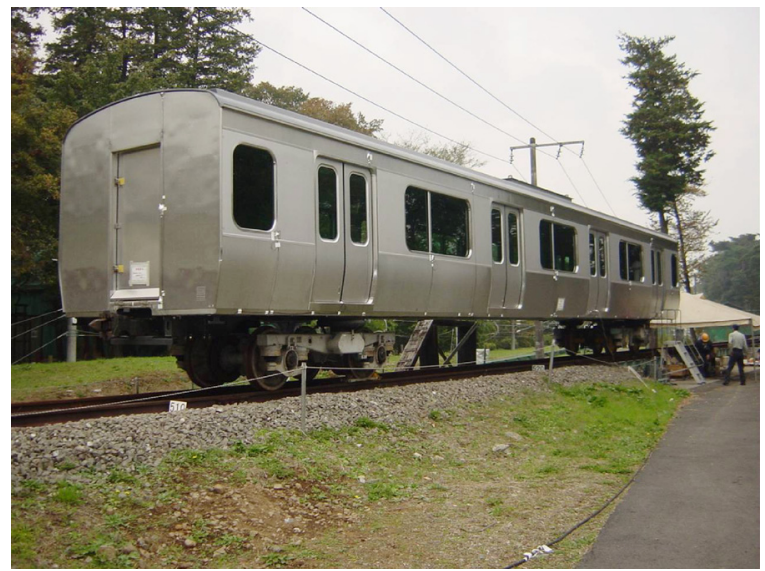

Fig. 1 Photo of commuter vehicle

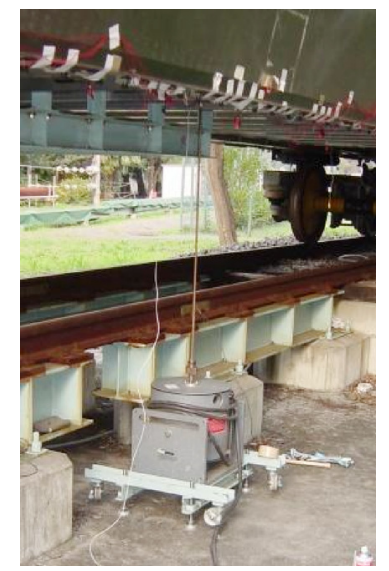

Fig. 2 Electrodynamic exciter

\section{Attachment of Piezoelectric Elements}

As described in the previous report (10), the authors carried out vibration suppression tests with piezoelectric elements using a 1:5 scale model of a Shinkansen. The length of the scale model was $4.9 \mathrm{~m}$ and the mass was about $292 \mathrm{~kg}$. In the previous test, eight pieces of 


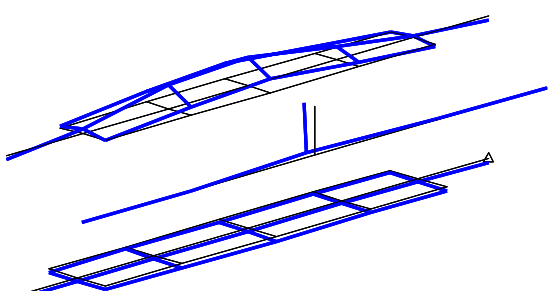

(a) $7.77 \mathrm{~Hz}$
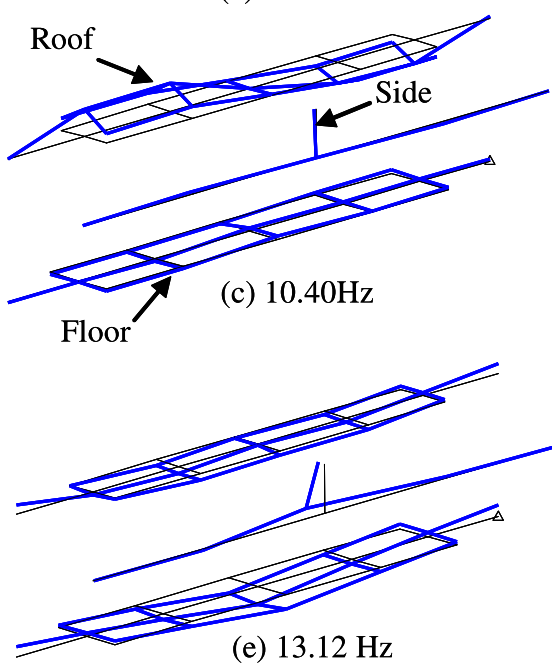

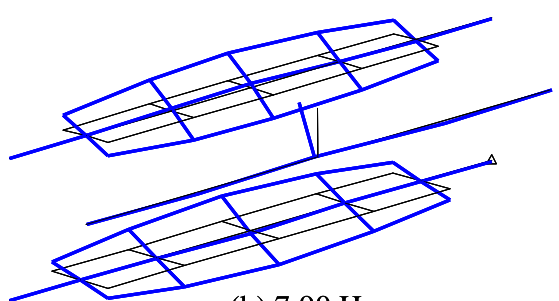

(b) $7.90 \mathrm{~Hz}$

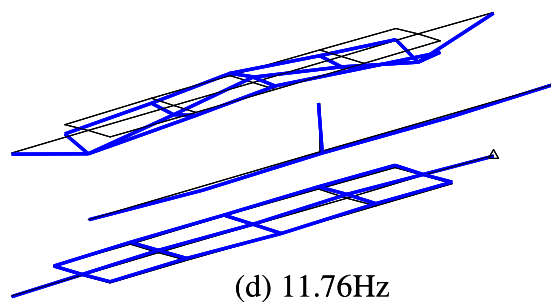

(d) $11.76 \mathrm{~Hz}$

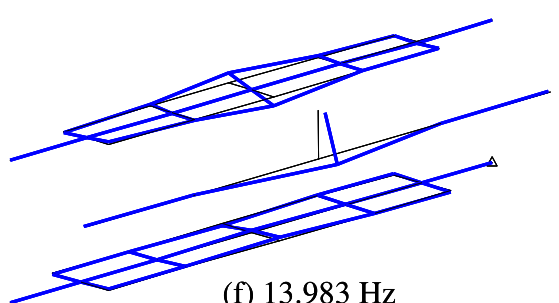

(f) $13.983 \mathrm{~Hz}$

Fig. 3 Modal characteristics of car body

piezoelectric elements, the size of each element was $155 \times 40 \times 3 \mathrm{~mm}$, were bonded at both side beams of the floor. On the other hand, the carbody used in this study is approximately four times larger in length and forty times heavier than the scale model. Therefore much more pieces of piezoelectric elements supposed to be needed. The PZT ceramics are employed as the piezoelectric elements in this study, because of high efficiency to generate voltage versus strain due to vibration, it is difficult to make very large size of ceramic pieces; therefore, it is unrealistic to make the size of PZTs larger according to the ratio of an actual carbody to the scale model.

In this study, we used the size of $155 \times 40 \times 2 \mathrm{~mm}$ PZTs, whose mass are approximately $140 \mathrm{~g}$ each, and they are almost the same size as used in the vibration suppression tests for the scale model. Since it is expected that relatively large number of such PZTs are needed, and considering the handling of the PZTs; attachment and/or detachment to carbody, ease in maintenance, ease to change the position of attachment etc., we develop the "Piezoelectric Damper Unit (PDU)" which consists of some pieces of PZTs and attachment jigs ${ }^{(11)}$. The PDUs are attached to the carbody by using some bolts.

In using the PDUs, there are some disadvantages from the aspect to transmit the carbody strain to the PZTs, by compared with the case to bond the PZTs directly. We made some trial types of jigs for PDUs, and compared the effectiveness of transmission of carbody strain, then decided the shapes of the jigs. Figure 4 shows the photo of the PZT, and Fig. 5 shows the two types of PDUs employed in this study.

It is effective to set the PDUs concentrically around where large carbody strain due to bending vibration is generated for vibration suppression. The type (A) PDU contains eight PZTs, layered by two pieces per set and four sets of laminated PZTs are slotted in three pieces of aluminum jigs. The type (A) PDUs are attached on the both side beams of the carbody floor using nine M6 bolts per unit. The number of piezoelectric elements is, however, shorter than demanded by using the type (A) PDUs. We therefore developed another type of PDU, type (B) shown in Fig. 5(b). The type (B) PDU contains $2 \times 2=4$ PZTs, and is attached between the cross beams with six M6 bolts. Two aluminum jigs in the type (B) PDUs are designed longer than that of type (A). Since the relative deformation of two adjoining cross 
beams is concentrated to the PZTs, it is expected that the strains in the PZTs increase and the performance of vibration suppression is improved.

In the excitation tests in the following section, the modes (b) and (e) are targeted to be suppressed, because vibration acceleration at the floor center around the natural frequencies of these two modes is conspicuous and it is assumable that these modes influence the ride comfort in the running conditions. The PDUs are arranged around the longitudinal center of the carbody because vibration shapes of both modes (b) and (e) show their loops at the position. The rows of type (A) and (B) PDUs are arranged parallel to each other, and attached to the carbody (See Fig.6). Numbers of the type (A) and (B) PDUs are 22 each, consisting of 264 PZTs. The total mass of PDUs including PZTs and the aluminum jigs is approximately $58 \mathrm{~kg}$, and the ratio to the carbody is approximately $0.55 \%$.

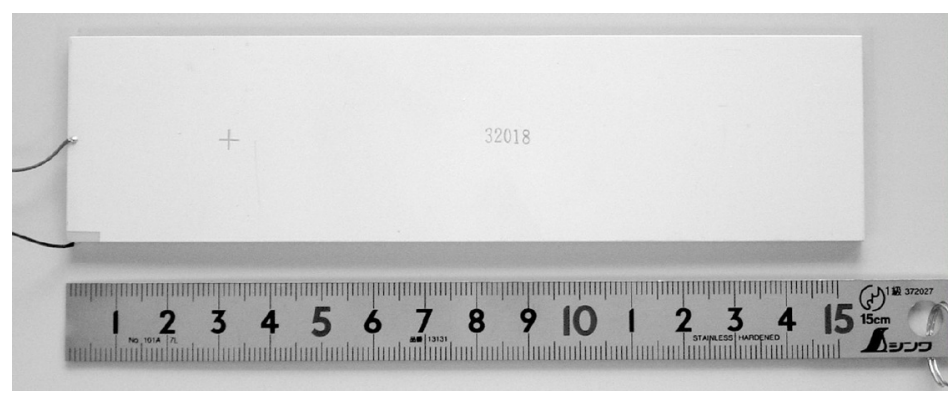

Fig. 4 Piezoelectric element (PZT)

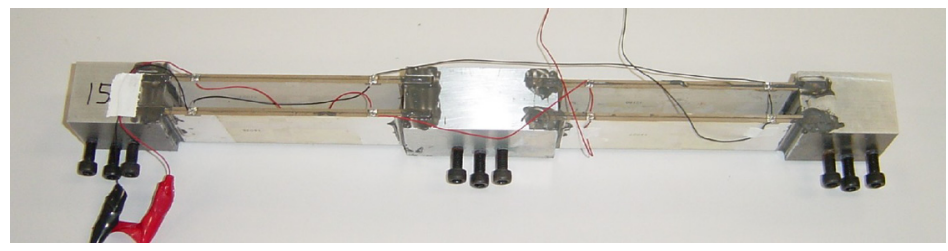

Type (A)

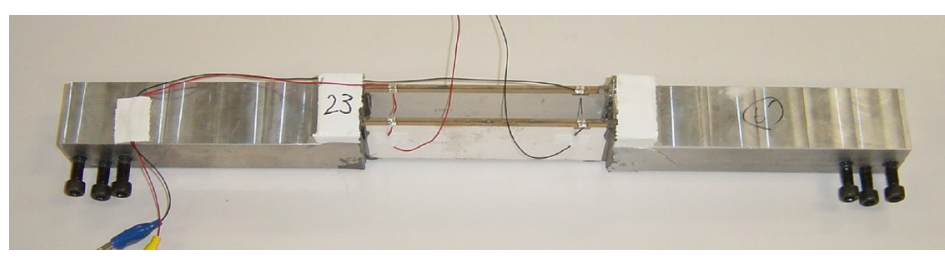

Type (B)

Fig. 5 Piezoelectric damper units (PDUs)

\section{Design of Electric Shunt Circuits}

\subsection{Types of Shunt Circuits}

Figure 7(a) is an electrically equivalent model of the piezoelectric element in the frequency range of interest ${ }^{(12)}$. It is composed of a capacitor $C_{p}$ and a voltage source $v_{p}$ due to the strain in the element.

An electric shunt circuit is connected to the electrodes of the elements in order to suppress the bending vibration of mechanical structures. Several types of shunt circuits have been proposed, and one of the most popular one, shown in the Fig.7(b), is an $L-R$ circuit, which consists of an inductor and a resistor in series. This type of shunt circuit suppresses the vibration more effectively than a resistor alone, because the $L-R$ circuit together with the inherent capacitor of the piezoelectric elements produce the damped electric resonance. The resonant frequency of the circuit is therefore tuned so that it is consistent with the natural frequency of the vibration mode of the mechanical structure to be suppressed. Therefore in 


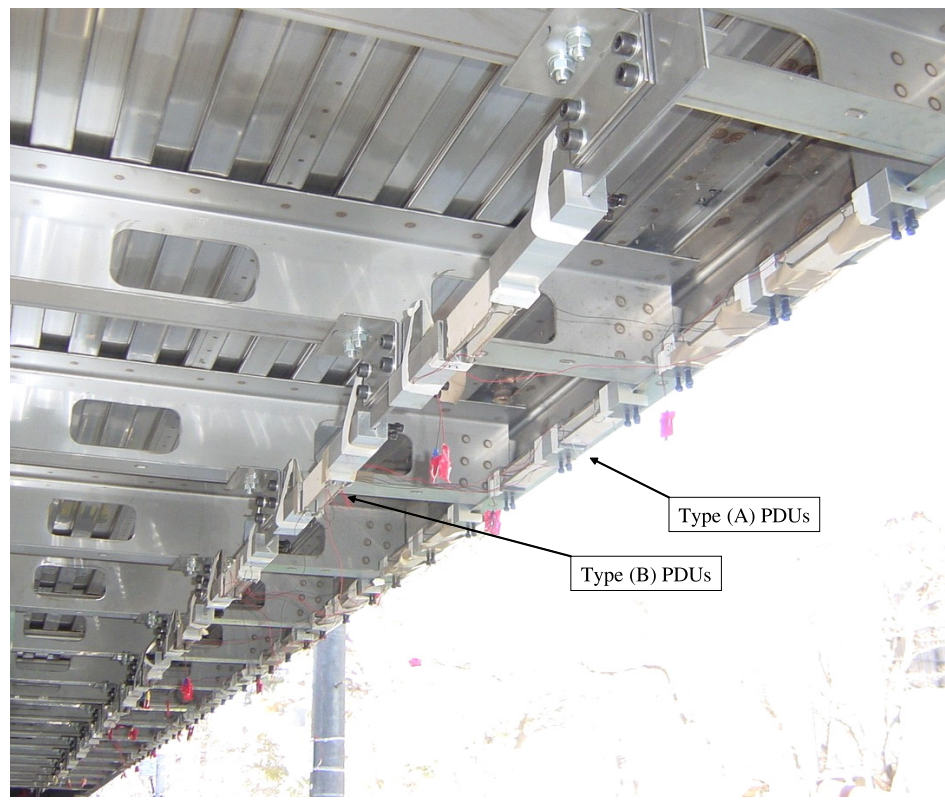

Fig. 6 PDUs attached to carbody

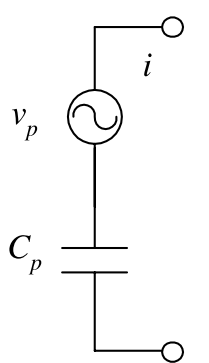

(a)Piezo. element

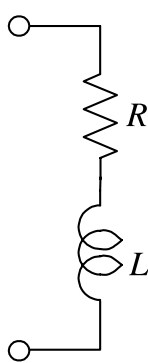

(b) $L-R$ circuit (c) Negative $C-R$ circuit

Fig. 7 Examples of shunt circuits

this case, the damping is effective against a single vibration mode.

On the other hand, another circuit, a negative $C-R$ circuit as shown in Fig.7(c) can realize multi-mode damping ${ }^{(13)}$. This circuit is composed of a capacitor with a negative value and a resistor in series. When the circuit is connected to the electrodes of the piezoelectric elements and the negative capacitor is tuned at $|C|=\left|C_{p}\right|$, it does not produce resonance, however, cancels the inherent capacitor directly and the total impedance of the circuit becomes only the pure resistance at all frequencies.

\subsection{Variable Inductor}

Although the $L-R$ circuit can be implemented only by the passive electric components, i.e. a coil and a resistor, a technique in which the characteristics of the inductor is synthesized by an active circuit including operational amplifiers (opamps) is widely utilized. It is because the optimal value of the inductor usually becomes too large to realize by the passive coil with ordinary size. Such the technique enables to vary and tune the inductance easily, however, it requires the electric power source and we can not make use of the advantages of the passive damping. In this study, therefore, a variable inductor composed of a passive coil is developed.

Optimal inductance $L_{o p t}$ can be calculated as:

$$
L_{o p t}=\frac{1}{C_{p}\left(2 \pi f_{n}\right)^{2}},
$$

where $f_{n}$ is the natural frequency of the structure to be suppressed. Therefore, $L_{o p t}$ can be kept 
small if one makes $C_{p}$ and $f_{n}$ large. Since the $f_{n}$, however, is not adjustable but a given value, we tried to enlarge the value of $C_{p}$ without influencing the mechanical characteristics of the piezoelectric elements.

A capacitance of a dielectric $C$ is generally expressible as follows:

$$
C=\frac{\epsilon A}{t},
$$

where $\epsilon$ is the permittivity, $A$ and $t$ are the area and the distance of the electrodes, respectively. This equation shows that $C_{p}$ can be enlarged by increasing the area, or by decreasing the thickness of the elements.

In the PDUs described in the previous section, two PZTs with a thickness of $2 \mathrm{~mm}$ are laminated instead of adopting one PZT with a thickness of 4mm. Although it is estimated that the both types of PZT(s) indicate almost identical mechanical characteristics in the longitudinal directions, the area and the thickness of the former are twice and 1/2 times as large as the latter, respectively, and the capacitance becomes four times resultantly. Additionally, it is known that the generated voltage by the strain in the elements is proportional to the $1 / C_{p}{ }^{(12)}$, therefore, this installation decreases the rated voltage of the coil to be designed.

The specifications of the variable inductor are determined as follows;

(1) Inductance is variable at least in the range of $0-20 \mathrm{H}$

(2) Inherent resistance is restricted to $2 \Omega / \mathrm{H}$ between 5 and $15 \mathrm{H}$

(3) Rated voltage is $150 \mathrm{~V}$

Spec.(1) is required to adapt the inductance to the changes of numbers of PZTs and vibration modes to be suppressed. Since it was verified in the previous study ${ }^{(10)}$ that the vibration suppression performance tends to deteriorate under the existence of surplus inherent resistance in the shunt circuit, the spec.(2) is provided. Spec.(3) is determined based on the results of numerical simulations in consideration of the running conditions with the parameters for an actual railway vehicle.

Figure 8 shows an outward appearance of the prototype of the variable inductor. The core of this inductor consists of two separated iron components and the aperture of them is variable by an adjusting handle. This mechanism enables to tune the value of inductance. The dimension of the inductor is 560 (width) $\times 560$ (depth) $\times 1000$ (height) $\mathrm{mm}$. The mass is $260 \mathrm{~kg}$ and ratio to the carbody is about $2.4 \%$, which is not too heavy compared with general railway equipments hung under the floor. Additionally note that the specifications listed above are determined for the experimental tests, and it is foreseeable that the mass could lighter in weight by limiting the functions.

\subsection{Implementation of Shunt Circuits Including Virtual Impedance}

The negative $C-R$ circuit with piezoelectric elements suppresses not only a single vibration mode but also multiple modes, while the $L-R$ circuit is generally effective for a single mode. The ride comfort will be improved with the $L-R$ circuit if one prominent eigen mode is dominant in the running conditions. Since it is reported, however, that not a few modes affect the ride comfort for the commuter vehicle, the Negative $C-R$ circuit is investigated in this section.

Since this type of circuit can not be implemented only by the passive electric components, appropriate active circuits should be designed. Figure 9 shows the generalized implementation of shunt circuits which the authors have proposed ${ }^{(14)}$.

In this circuit, one of the electrodes of the piezoelectric element is connected to impedance components $Z_{0}$ and $Z_{i}$ in series. $v_{0}$ and $v_{i}$ are voltage applied to these components. The voltage $v_{i}$ is input into an amplifier $G(s)$, and amplified to voltage $V_{\text {out }}(s)=G(s) V_{i}(s)$, where capital letters followed by $(s)$ represent the Laplace Transform of corresponding variables or transfer functions. Note that $G(s)$ can realized by not only an analog electric circuit, but also a digital controller accompanied by a piezoelectric driver. The output voltage $v_{\text {out }}$ is applied to the other electrode of the element. 


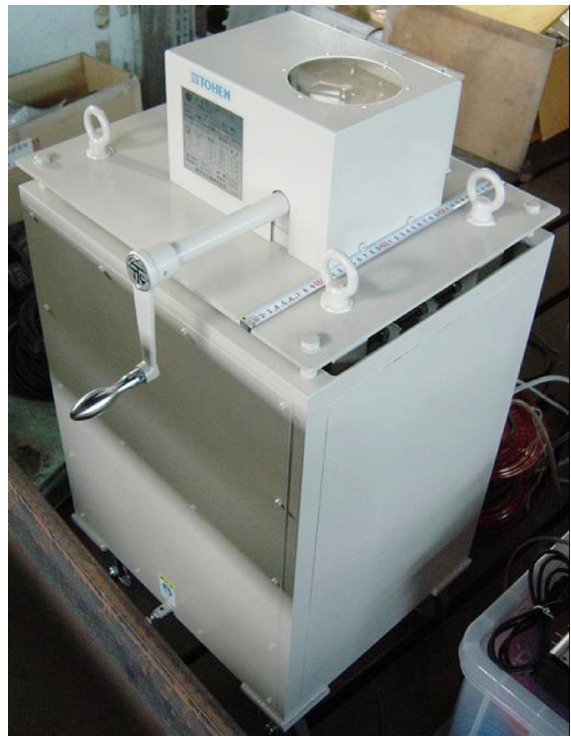

Fig. 8 Variable inductor

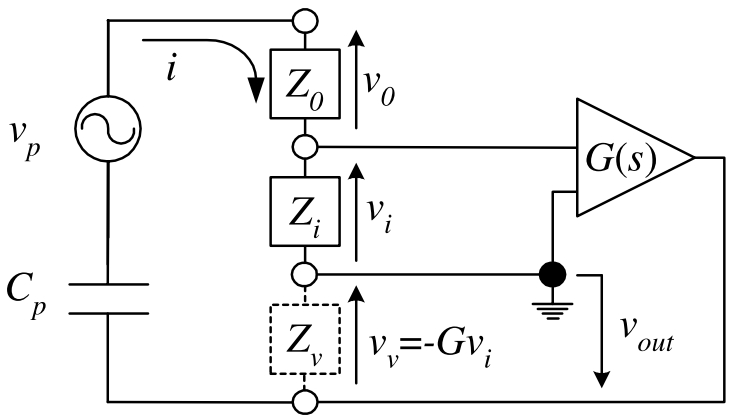

Fig. 9 Proposed implementation of shunt circuits including virtual impedance $Z_{v}$

In such a composition, the ratio of two voltages $v_{0}$ and $v_{i}$ in the Laplace domain becomes:

$$
\frac{V_{i}(s)}{V_{0}(s)}=\frac{Z_{i}(s)}{Z_{0}(s)}
$$

because a common current $i$ flows through these two impedance components. Another relation of voltages can be obtained as:

$$
\frac{V_{v}(s)}{V_{i}(s)}=\frac{-G(s) Z_{i}(s)}{Z_{i}(s)}
$$

where $v_{v}=-v_{\text {out }}$. Therefore, it is expected that the proposed circuit in Fig. 9 shows the same behavior as that of serially connected three components, $Z_{0}(s), Z_{i}(s)$ and $-G(s) Z_{i}(s)$. This fact indicates that the virtual impedance,

$$
Z_{v}(s)=-G(s) Z_{i}(s)
$$

is produced. In this way, the total impedance is realized as:

$$
Z_{s}(s)=Z_{0}(s)+Z_{i}(s)+Z_{v}(s)=Z_{0}(s)+(1-G(s)) Z_{i}(s) .
$$

Consequently, when the total impedance $Z_{s}=Z_{o p t}$ to be realized is determined, the actual impedance components $Z_{0}$ and $Z_{1}$ are chosen first, and then the transfer function $G(s)$ is designed as:

$$
G(s)=\frac{Z_{i}(s)+Z_{0}(s)-Z_{o p t}(s)}{Z_{i}(s)} .
$$

Note that both $Z_{0}$ and $Z_{i}$ are mounted as actual (passive) electric parts, but only $Z_{i}$ influences the characteristics of virtually realized impedance $Z_{v}$. 
This implementation will be utilized to produce a negative resistor in $\S 5.1$ and a negative capacitor in $\$ 5.2$.

\section{Excitation Tests}

The PDUs described in the $\S 3$ are attached under the floor around longitudinal center. The PZTs are shunted by the $L-R$ or the negative $C-R$ circuit. The carbody is excited by the electrodynamic exciter with the band random signal, which is the same as the signal used in the preliminary tests, and the frequency response function (FRF) between excitation force and the acceleration on the floor above the excitation point is calculated to examine the validity of the proposed method.

Figures 10 and 11 show the FRFs between the excitation force and the acceleration on the floor at the longitudinal center near the side window. These figures show the same FRFs but the Figs.11(i) and 11(ii) are focused on the frequency ranges around the natural frequencies of the modes (e) and (b), which are the targeted modes to be suppressed, respectively. Black lines in these figures show the FRF when all electrodes are short-circuited, i.e., no electric damping effects are appended. Note that the FRFs in the Figs.11(i) and 11(ii) are normalized by the focused natural frequencies and the respective peak values of the short-circuited case. The numbers as shown in the legend express the ratios of the peak values for respective conditions to that of the short-circuited case.

\subsection{Results with $L-R$ Circuit}

The $L-R$ circuit is implemented by the variable inductor shown in the $\S 4.2$. As mentioned previously, this circuit is effective for a single vibration mode because the optimal values of inductance $L_{o p t}$ are different for the modes (e) and (b). These modes, therefore, cannot be targeted simultaneously and two separate excitation tests are performed targeted on each of the modes (e) and (b), by setting the different $L_{o p t}$ for respective modes.

All electrodes of the PZTs attached on the left side beam are connected in parallel, and so as the PZTs on the right side beam. The two assembled electrodes (left and right) are then connected. The polarities of these electrodes are the same when the target mode is (e), and reversed for the mode (b). It is because the all generated voltage of PZTs are in phase when the carbody is vibrating with the mode (e), while the voltages of PZTs on the left and right sides are antiphase when the mode (b) is excited. Total capacitance of the PTZs is $C_{p}=10.4 \mu \mathrm{F}$, which is measured experimentally, and the value is the same for both connections.

Blue lines in the Figs. 10 and 11 show the FRF with an $L-R$ circuit, in which $L$ is tuned so as to suppress the mode (e). The optimal value of inductance in this case is set as $L=14.1 \mathrm{H}$, which is calculated based on the Eq.(7) with the measured $C_{p}$ and $f_{n}$. No resistor $R$ as an actual component is installed because the variable inductor includes the inherent resistance. The FRF peak at the natural frequency of the mode (e) is reduced by 19\% (See Fig.11(i)), however, no vibration suppression is observed at the natural frequency of the mode (b) (Fig.11(ii)) when this $L-R$ circuit is connected.

The light blue lines in the Figs.10 and 11 show the FRF with another $L-R$ circuit in which the inductance is set as $L=39.0 \mathrm{H}$ and the mode (b) is targeted. The FRF peak value at the natural frequency of the mode (e) is almost the same with that of the short-circuited case, while the peak is reduced by $12 \%$ with this $L-R$ circuit at the natural frequency of the mode (b) (See Fig.11(ii)). The performance, however, in this case is a little poorer than that when the mode (e) is targeted. The shape of the FRF implies that the resistor in the shunt circuit is too large, because no antiresonance appears in the FRF.

Actually, the $L=39.0 \mathrm{H}$ is out of the ranges in the spec.(1) and (2) of the variable inductor, listed in the $\$ 4.2$. Although inductance value is realized practically, the inherent resistance exceeds the limit of the spec.(2), and this possibly lead to the relatively poor performance.

Therefore, a resistance with negative value is then tried to be appended to the variable inductor in order to reduce the inherent resistance in the circuit. By setting the parameters in 


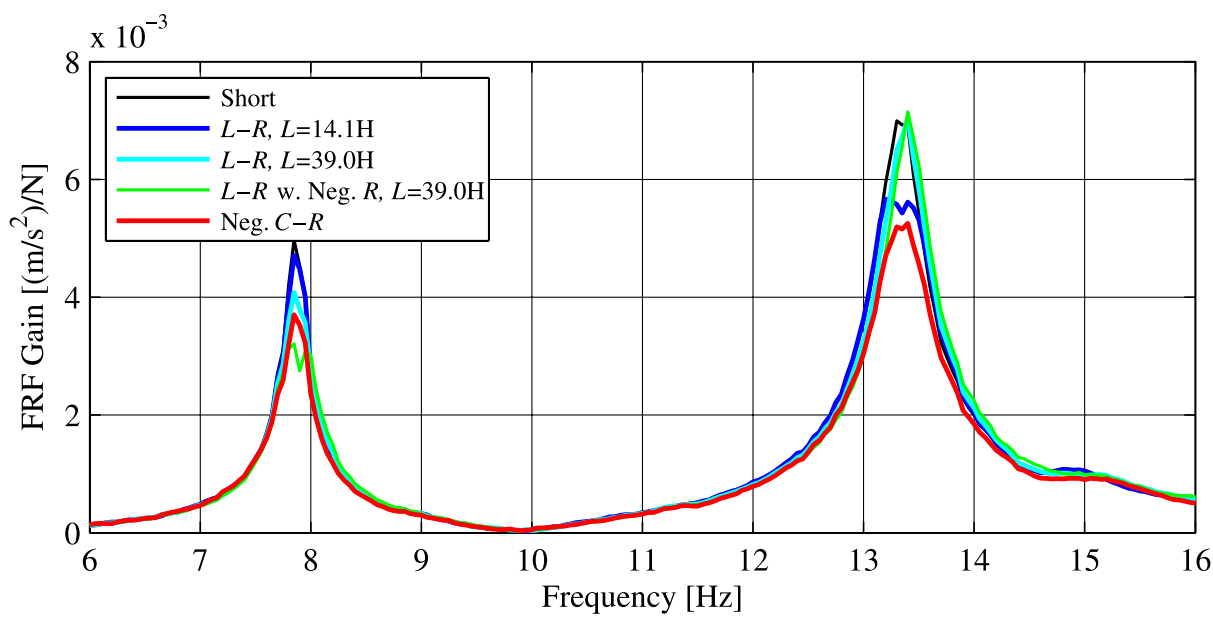

Fig. 10 Measured FRFs between excitation force and acceleration on floor

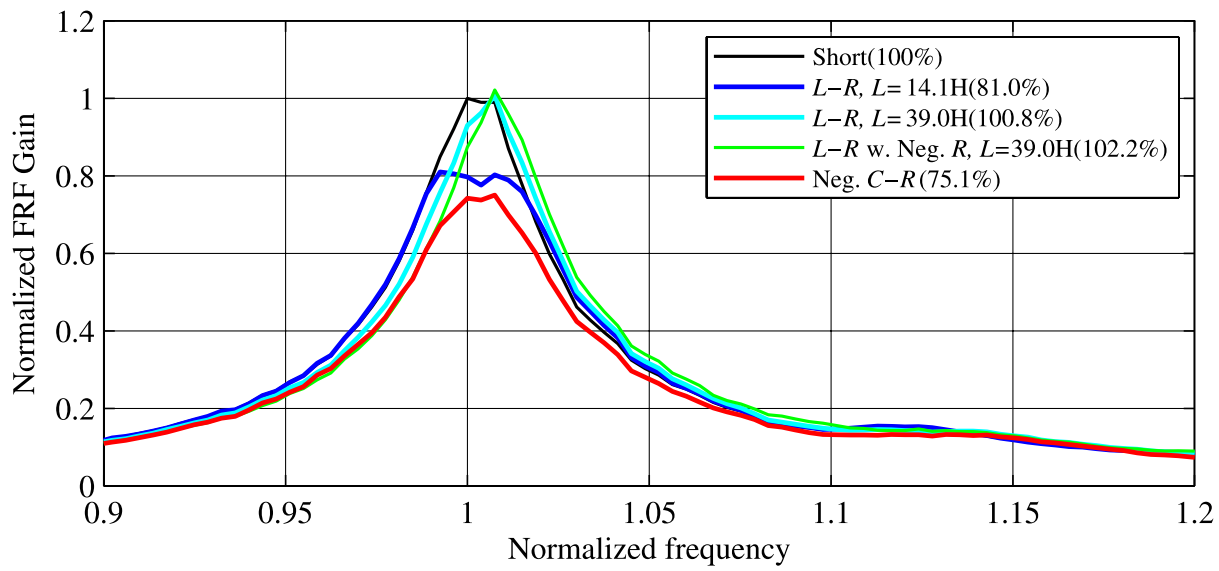

(i) Around natural frequency of mode (e)

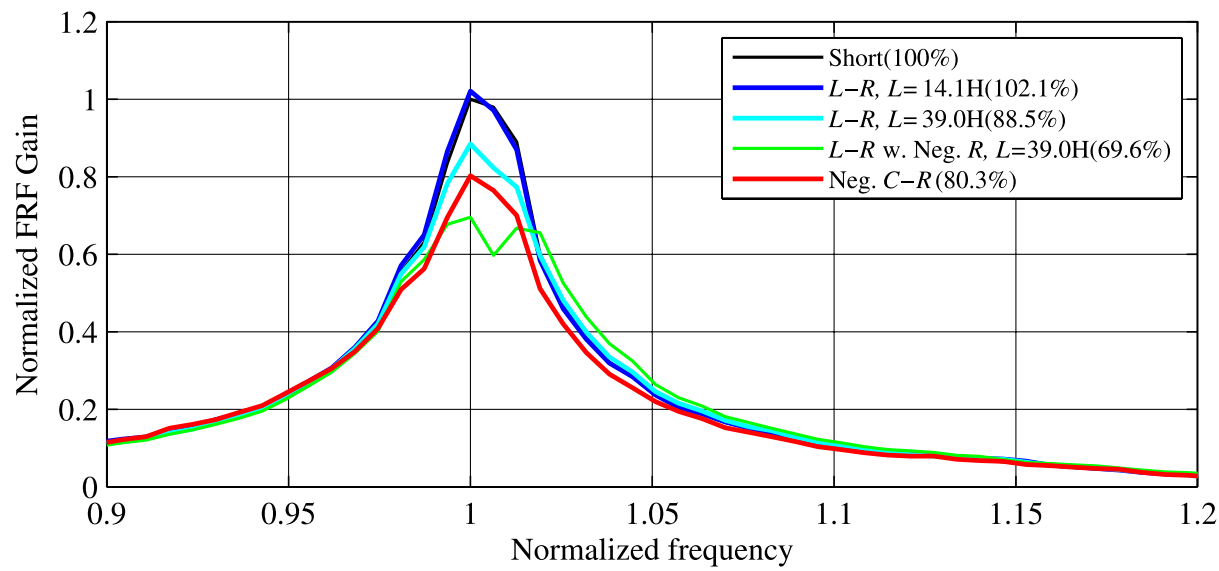

(ii) Around natural frequency of mode (b)

Fig. 11 Normalized FRFs between excitation force and acceleration on floor

the Fig.9 as follows;

$$
\begin{aligned}
& Z_{0}(s)=Z_{l} \text { (Impedance of variable inductor) } \\
& Z_{i}(s)=R_{i}=40 \text { (Resistor as actual component) } \\
& G(s)=2.0,
\end{aligned}
$$

the virtual negative resistance $Z_{v}=-80 \Omega$ is produced in the circuit, according to the Eq.(5). This circuit consequently adds the total resistance in the shunt circuit by $Z_{v}+Z_{i}=-40 \Omega$. The FRF with this circuit is shown in the green lines in the Figs.10 and 11. The FRF peak value at the natural frequency of the mode (b) is reduced by $30 \%$ and the performance of the vibration 
suppression is improved by reducing the inherent resistor.

\subsection{Results with Negative $C-R$ Circuit}

The negative $C-R$ circuit is implemented based on the Fig.9. The modes (b) and (e) are targeted simultaneously in this section, because this circuit can realize the multi-mode damping. Two negative $C-R$ circuits, one is connected to the PZTs on left side beams, and the other is to the right, are composed.

The parameters are the same for these two circuits and determined as follows:

$$
\begin{aligned}
& Z_{0}(s)=0 \text { (Short-circuited) } \\
& Z_{i}(s)=R_{i}=100 \text { (Resistor as actual component) } \\
& G(s)=-\frac{1}{\alpha s}-\beta+1 .
\end{aligned}
$$

The total impedance of each shunt circuit is

$$
\begin{aligned}
Z_{i}(1-G(s)) & =R_{i}-R_{i}\left(-\frac{1}{\alpha s}-\beta+1\right) \\
& =\frac{1}{\left(\alpha / R_{i}\right) s}+\beta R_{i} .
\end{aligned}
$$

This impedance is equivalent to that of a capacitor whose capacitance is $C_{v}=\alpha / R_{i}$ and a resistor whose resistance is $R_{v}=\beta R_{i}$ in series. The virtually produced capacitance is set as $C_{v}=-C_{p} / 2$ because one negative $C-R$ circuit is used for a half of total numbers of PZTs. The virtual resistance $R_{v}$ is set as a negative value because it is expected that the reduction of the total resistance improves the performance of the vibration suppression, according to the results in the previous section (Fig.11(ii)). Since it is difficult, however, to estimate the optimal value of $R_{v}$ theoretically, this value is tuned during the excitation tests and fixed as $R_{v}=-100 \Omega$.

The transfer function $G(s)$ with a bandpass filter is discritized at a sampling period of $2 \mathrm{kHz}$ and implemented in a PC as a digital filter. The bandpass filter is appended to remove the DC offset voltage and to avoid the high transfer gain in the high frequency range. The FRF with this circuit is shown in red lines in the Figs.10 and 11 . Note that the both red lines in the Figs.11(i) and 11(ii) (for negative $C-R$ circuit) are calculated from the measured data in one excitation test, while the blue and light blue lines (for $L-R$ circuits with different values of $L$ ) are the results of two separate excitation tests because the optimal values of $L$ for both tests are different. These FRFs show that the peak values related to the respective modes (e) and (b) are simultaneously reduced by 25 and $20 \%$, respectively, with the negative $C-R$ circuit as compared with the short-circuited case.

\section{Conclusions}

The vibration suppression technique that utilizes piezoelectric elements shunted by external electric circuits is applied to reduction of bending vibration of an actual railway vehicle carbody. We investigated the attachment devices and the design strategy of shunt circuits in this study, aiming at the practical use.

The piezoelectric damper units (PDUs), consisting of PZTs and attachment jigs made of aluminum alloy, are developed. The PDUs are fixed to the side beams of the carbody with bolts. $L-R$ and a negative $C-R$ circuits as a shunt circuits are tested in the excitation tests. The $L-R$ circuits are implemented by the variable inductor composed of a passive coil, and the negative $C-R$ circuit is designed based on the originally proposed method, in which the part of the impedance characteristics are virtually produced by the software procedures.

We performed stationary excitation tests by using a commuter vehicle whose length is approximately $20 \mathrm{~m}$ and the frequency response function (FRF) between the excitation force and accelerations on the carbody is evaluated. The FRFs with the $L-R$ or the negative $C-R$ circuits are reduced up to $30 \%$ compared with the case where the PZTs are short-circuited, i.e., no electric damping effects are appended. 
Next steps of our study are to make the PZTs provide shock and water resistances, and to improve the performance of vibration suppression. The authors are planning to carry out the excitation tests under the conditions which are equivalent to the running conditions in the near future, and are investigating this vibration technique aiming at putting it to practical use.

\section{References}

( 1 ) Suzuki, Y., Akutsu, K., Maebashi E. and Sasakura, M., Method for Flexural Vibration Damping for Rolling Stock carbody, Quarterly Report of RTRI, Vol.38, No.3(1997), pp. 123-128.

( 2 ) Tomioka, T. and Takigami, T., Suppression of Flexural Vibration of Railway Vehicle Carbody by Using Carbody-Truck Interaction, Transactions of the Japan Society of Mechanical Engineers, Series C, Vol.70, No.696(2004), pp.2419-2426 (in Japanese).

( 3 ) Nagai, M. and Sawada, Y., Active Suspension Control for the Flexible Structure of an Elastic Vehicle Body, Transactions of the Japan Society of Mechanical Engineers, Series C, Vol.53, No.492(1987), pp. 1750-1757 (in Japanese).

( 4 ) Sugahara, Y., Takigami, T. and Sampei, M., Suppressing Vertical Vibration in Railway Vehicles through Primary Suspension Damping Force Control, Journal of System Design and Dynamics, Vol.1, No.2(2007), pp.224-235.

( 5 ) Hagood, N. W. and Flotow, A. v, Damping of Structural Vibration with Piezoelectric Materials and Passive Electrical Networks, Journal of Sound and Vibration, Vol.146, No.2(1991), pp. 243-268.

( 6 ) Lesieutre, G. A., Recent Advances in the Use of Piezoceramics for Vibration Suppression, The Shock and Vibration Digest, Vol.30, No.3, (198), pp. 187-195.

( 7 ) Fujita, T., Nomura, H., Yasuda, M., Matsuura, A. and Tchuchiya, M., Fundamental Study of Passive Microvibration Control with Smart Structure using Piezoelectric Devices, Transactions of the Japan Society of Mechanical Engineers, Series C, Vol.66, No.644(2000), pp. 1097-1101 (in Japanese).

( 8 ) Ho, S. T, Matsuhisa, H., and Honda, Y., Passive Vibration Suppression of Beam with Piezoelectric Elements (An Approach Using Analogies between Electrical and Mechanical Circuits), JSME International Journal, Series C, Vol.43, No.3(2000), pp. 740-747 (in Japanese).

( 9 ) Takigami, T. and Tomioka, T., Passive Vibration Suppression of Railway Carbody with Piezoelectric Elements (Feasibility Study by Using Pipe Model), Transactions of the Japan Society of Mechanical Engineers, Series C, Vol.68, No.674(2002), pp. 3029-3036 (in Japanese).

(10) Takigami, T., Tomioka, T. and Hansson, J., Vibration Suppression of Railway Vehicle Carbody with Piezoelectric Elements (A Study by Using a Scale Model of Shinkansen), Journal of Advanced Mechanical Design, Systems, and Manufacturing, Vol.1, No.5(2007), pp649-660.

(11) Miyata, S., Nakano, S., Suzuki, K., Takigami, T., Tomioka, T. and Suzuki, Y., Vibration Suppression of Railway Carbody with Piezoelectric Elements (Attachment Method of Element for Damping Performance Improvement), Proceedings of Mechanical Engineering Congress, the Japan Society of Mechanical Engineers, Vol.6(2005-9), pp175176 (in Japanese).

(12) Dosch, J., Inman, D., A Self-Sensing Piezoelectric Actuator for Collocated Control, Journal of Intelligent Material Systems and Structures No.3(1992), pp.166-185.

(13) Behrens, S., Fleming, A.J. and Moheimani, S.O.R., A Broadband Controller for Shunt Piezoelectric Damping of Structural Vibration, Smart Materials and Structures, Vol.12, No.1(2003), pp. 18-28.

(14) Takigami, T. and Tomioka, T., Vibration Suppression of Railway Carbody with Piezoelectric Elements (A Study Focused on Designing Shunt Circuits), Proceedings of SPIE, Smart Strucures and Materials, Vol.5760(2005-3), pp. 329-340. 